\title{
Electrostatic Microactuator Design Using Surface Acoustic Wave Devices
}

\author{
Don W. Dissanayake, Said F. Al-Sarawi and Derek Abbott
}

\begin{abstract}
An integration of low power operated Surface Acoustic Wave (SAW) devices with the electrostatic microactuators for microfluidic and similar applications is presented in this chapter. Passive, low power, and small area devices can be interrogated wirelessly using SAW devices, which can respond to a uniquely coded signal for a secure and reliable operation. The novel approach relies on converting the interrogating coded signal to surface acoustic wave that is then correlated with an embedded code. A theoretical analysis of how the actuation mechanism operates is carried out and simulation results of the new microactuator are discussed. At the initial analytical stage, for simplicity, a basic SAW delay line structure is used to generate an electrostatic field between output interdigital transducer (IDT) of the SAW device and a thin conductive plate (actuator), which is placed on top of the output IDT. The static and transient displacement analysis of the actuator is carried out using ANSYS simulation tools. A comparison between the static displacements obtained from ANSYS based simulations and Rayleigh-Ritz based analysis is also presented and discussed.
\end{abstract}

Keywords SAW device · wireless · microactuator $\cdot$ electrostatic $\cdot$ IDT $\cdot$ ANSYS

\footnotetext{
Don W. Dissanayake

Centre for High Performance Integrated Technologies and Systems (CHiPTec), School of Electrical and Electronic Engineering, University of Adelaide, Australia, e-mail: don@eleceng.adelaide.edu.au

Said F. Al-Sarawi

Centre for High Performance Integrated Technologies and Systems (CHiPTec), School of Electrical and Electronic Engineering, University of Adelaide, Australia, e-mail: alsarawi@eleceng.adelaide.edu.au

Derek Abbott

Centre for Biomedical Engineering (CBME), School of Electrical and Electronic Engineering, University of Adelaide, Australia,

e-mail: dabbott@eleceng.adelaide.edu.au
} 


\section{Introduction}

Micro Electro Mechanical Systems (MEMS) based microvalves and micropumps are of greater interest when it comes to nanolitre drug delivery and similar biomedical applications. Actuators are one of the important components in microvalves and micropumps for fluid manipulation. The design of a suitable actuator device to modulate the fluid at the microscale, for accurate operation, is of great importance. Many types of microactuators have been developed to match different requirements for various applications $[1,2]$. With miniaturization, physical scaling laws inherently favour some technologies and phenomena over others. In some cases, technologies that can be made by micromachining work well at the microscopic scale, but have no analogy or usefulness in the macroscopic domain. Moreover most of these actuators are too complicated to fabricate within a micropump structure.

Notably SAW devices are used to develop micromachines such as ultrasonic micromotors and fluid transfer methodologies such as flexural micropumps [3, 4]. Currently available microfabrication technologies such as photolithography and X-ray lithography with a combination of other processes have enabled the use of SAW devices for a variety of self-contained MEMS applications, which have advanced functionality and performance. The key benefits of these micromachines are in their small size, ease of production, and low-cost.The use of SAW devices for micro actuation applications provides the great benefit of controlling and interrogating the devices remotely without direct user intervention $[1,2,5]$.

In this chapter, SAW based electrostatic actuator modeling is discussed. Consequently such a microactuator can be embedded in a microfluidic device to modulate the fluid flow using less power. In Sect. 2, the use of SAW devices for micro actuation is discussed. Section 3 explains the operation of the SAW device based microactuator. An underlying theoretical model is then elaborated in Sect. 4 and followed by a method to derive the electric potential wave at the output IDT in Sect. 5. Section 6 shows the boundary condition analysis for the proposed model. Section 7 discusses the simulation and results and is followed by a conclusion.

\section{SAW Based Microactuator}

SAW device related technology has been utilised to design and develop MEMS based microaccelerometers and gyroscopes for military and similar applications $[2,6]$. The technology used in those applications is similar to the capacitor effect generated by programmable tapped delay lines, which use the principle of air gap coupling [7] between the SAW substrate and a silicon superstrate. These capacitors are then used to control the amount of Radio Frequency (RF) coupling from the input IDT on the SAW substrate to the output terminal on the silicon chip [6]. It is a well known method to use a sandwich structure of semiconductor on piezoelectric substrate to form so called space-charge coupled SAW devices and SAW convolvers [7]. 


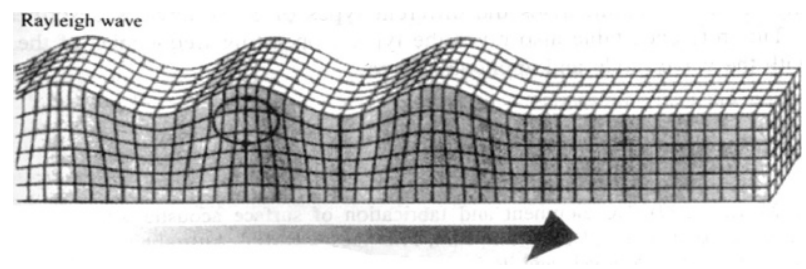

Fig. 1 Rayleigh Wave Mode which shows elliptical particle displacements [8]

Such an approach can be utilised in the design of a SAW based microactuator. The proposed approach for the actuator design is converse to the method used in [6] for the microaccelerometer design. Being an elastic deformation wave on a piezoelectric substrate, the SAW induces charge separation. Thus it carries an electric field with it, which exists both inside and outside the piezoelectric substrate and decays according to Laplace's equation. In this SAW device based actuator, a thin conductive plate is placed on top of the output IDT, which is separated by an air-gap. The conductive plate does not alter the mechanical boundary conditions of the SAW substrate, but causes the surface to be equipotential and the propagating electric potential to be zero at the surface of the conductive plate. As a result an electrostatic force is generated between the conductive plate and the output IDT in the SAW device causing micro deformations in the conductive plate.

It is highly important to choose a suitable SAW mode for the SAW device especially when it is designed for microfluidic applications. Therefore, different acoustic modes need to be analysed for example, one would expect horizontal shear SAW mode to be more useful than Rayleigh SAW mode. This is because when a SAW device is in vitro, Rayleigh SAW mode is rapidly damped out, due to the pressure generated by the surrounding fluid [5]. In contrast to the general approach of using the SAW device surface to transfer the fluid, the proposed approach uses an actuator (conductive plate), which is on top of the SAW substrate. As a result the fluid flow is isolated from the SAW substrate and hence it prohibits the interaction of the fluid with the SAW mode. Moreover, Rayleigh SAW mode is best suited for space-charge related applications as most of the energy in this mode is concentrated within one wavelength of the substrate [7], as shown in Fig. 1. Hence, considering the measures taken to isolate the SAW substrate from the fluid in this proposed approach, Rayleigh SAW mode is chosen.

\section{Proposed Microactuator Operation}

A SAW substrate made out of 128-YX-Lithium Niobate and a conductive plate made out of silicon are used to generate an air gap coupled SAW based electrostatic actuator. The device operation is as follows. The input IDT generate Rayleigh waves using inverse piezoelectric effect based on the RF signal that is being fed to the SAW device through the microstrip antenna. The Output IDT regenerates the 
electric signal using the piezoelectric effect of the SAW device. As it was explained in Sect. 2, the generated electrostatic field between this propagating electric potential wave and the conductive plate on top of the output IDT creates a compulsive and repulsive force between the two. Since the conductive plate is a thin flexural plate, it bends as a function of the applied electrostatic field enabling its use as a microactuator.

\section{Theoretical Model}

In piezoelectric materials, the frequency of the induced electric field wave is sufficiently small enough so that the electromagnetic coupling effects can be safely discarded. This means local perturbations are felt almost instantaneously through out the substrate, hence the electric field can be treated as quasi-static $[9,10,11]$. In the piezoelectric analysis this is achieved by setting the permeability to zero, corresponding to an infinite speed of the electromagnetic wave. Using Maxwell's equations, electromagnetic quasi static approximation can be written as

$$
\begin{gathered}
\mathbf{E}_{i}=-\frac{\partial \Phi}{\partial x_{i}}, \\
\nabla \cdot \mathbf{D}=0,
\end{gathered}
$$

where $\mathbf{E}$ is the electric field, $\Phi$ is the electric potential, $\mathbf{D}$ is the electric displacement and $i=1,2,3$ shows the direction of interest as shown in Fig. 2. From the mechanical equations of motion,

$$
\rho \frac{\partial^{2} \mathbf{u}_{i}}{\partial t^{2}}=\sum_{j} \frac{\partial \mathbf{T}_{i j}}{\partial x_{i}},
$$

where $\rho$ is the density of the piezoelectric substrate, $\mathbf{u}$ is the mechanical displacement and $\mathbf{T}$ is the mechanical stress.

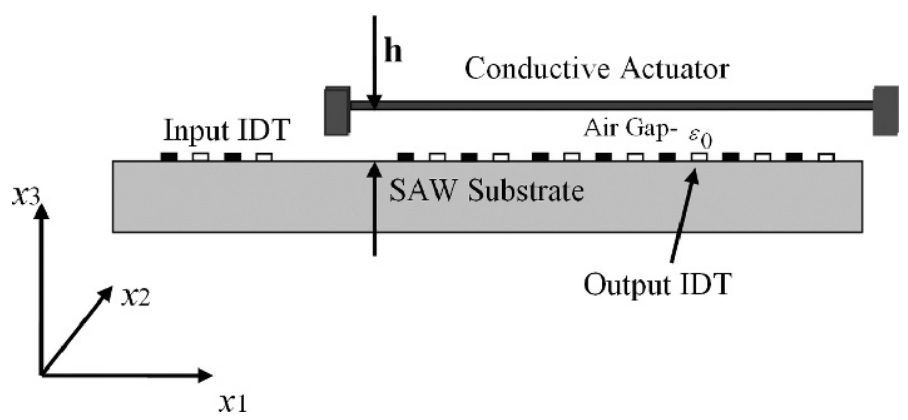

Fig. 2 SAW Actuator model and boundary conditions. The SAW substrate is the rotated crystal cut to make sure the wave propagation in $x_{1}$ direction and $h$ is the height of the air gap 
The relation between the mechanical strain $\mathbf{S}$ and mechanical displacement can be written as

$$
\mathbf{S}_{i j}=\frac{1}{2}\left[\frac{\partial \mathbf{u}_{i}}{\partial x_{j}}+\frac{\partial \mathbf{u}_{j}}{\partial x_{i}}\right] .
$$

Constitutive equations for piezoelectric material give the relationship between $\mathbf{D}$, $\mathbf{E}, \mathbf{T}$ and $\mathbf{S}$ in a SAW substrate as

$$
\begin{aligned}
\mathbf{T}_{i j} & =\sum_{k} \sum_{l} \mathbf{c}_{i j k l}^{E} \mathbf{S}_{k l}-\sum_{k} \mathbf{e}_{k i j} \mathbf{E}_{k}, \\
\mathbf{D}_{i} & =\sum_{j} \sum_{k} \mathbf{e}_{i j k} \mathbf{S}_{j k}+\sum_{j} \varepsilon_{i j}^{S} \mathbf{E}_{j},
\end{aligned}
$$

where $\mathbf{c}$ is the stiffness tensor for constant electric field, $\mathbf{e}$ is the piezoelectric coupling tensor, $\varepsilon$ is the permittivity tensor for constant strain and $i, j, k, l$ go from 1 to 3 . Equations (1-6) leads to a system of four coupled equations, which are represented by (7) and (8) below.

$$
\begin{gathered}
\sum_{j} \sum_{k}\left[\mathbf{e}_{k i j} \frac{\partial^{2} \Phi}{\partial x_{j} \partial x_{k}}+\sum_{l} \mathbf{c}_{i j k l}^{E} \frac{\partial^{2} \mathbf{u}_{k}}{\partial x_{j} \partial x_{l}}\right]=\rho \frac{\partial^{2} \mathbf{u}_{i}}{\partial t^{2}} \\
\sum_{i} \sum_{j}\left[\varepsilon_{i j}^{S} \frac{\partial^{2} \Phi}{\partial x_{i} \partial x_{j}}-\sum_{k} \mathbf{e}_{i j k} \frac{\partial^{2} \mathbf{u}_{j}}{\partial x_{i} \partial x_{k}}\right]=0 .
\end{gathered}
$$

This relationship between mechanical displacement and electric potential is considered in a semi-infinite, isotropic and homogenous linear elastic space as shown in Fig. 1. The crystal cut ensures the Rayleigh wave propagate in the $x_{1}-x_{3}$ sagittal plane as in Fig. 2. Sagittal plane is the plane defined by the real wave vector (wave number) $k$ and the unit vector normal to the surface of the SAW substrate ( $x_{3}$ direction). The method of partial waves can be used to solve the problem of wave propagation on anisotropic substrates such as piezoelectrics [9, 12]. As a result, plane wave solutions of the form in equations (9) and (10) are considered for the mathematical modeling of this device,

$$
\begin{gathered}
\mathbf{u}_{j}^{m}\left(x_{1}, x_{3}, t\right)=\alpha_{j}^{m} e^{\mathrm{i} k b^{m} x_{3}} \cdot e^{\mathrm{i} k\left(x_{1}-v t\right)}, \\
\boldsymbol{\Phi}^{m}\left(x_{1}, x_{3}, t\right)=\alpha_{4}^{m} e^{\mathrm{i} k b^{m} x_{3}} \cdot e^{\mathrm{i} k\left(x_{1}-v t\right)},
\end{gathered}
$$

where the $\alpha_{j}$ values are linear coefficients that depend on the decaying constant $b, v$ is the SAW velocity in the substrate, $m=1,2,3,4$ and $j=1,2,3$.

By substituting the plane wave solutions given in (9) and (10) into the coupling wave equations (7) and (8), a linear system for the coefficients $\alpha_{j}$ can be obtained and solved. A similar approach has been adopted in microaccelerometer modelling in [6]. Following the approach discussed in [6], a general solution is obtained and the resulting equations can be seen from (11) and (12). The weighting coefficients 
C of these plane waves are chosen to satisfy the mechanical and electrical boundary conditions at the surface of the piezoelectric substrate specific to this SAW based actuator model is presented in Sect. 6,

$$
\begin{aligned}
& \mathbf{U}_{j}\left(x_{1}, x_{3}, t\right)=\left[\sum_{m} \mathrm{C}_{m} \alpha_{j}^{m} e^{\mathrm{i} k b^{m} x_{3}}\right] \cdot e^{\mathrm{i} k\left(x_{1}-v t\right)}, \\
& \boldsymbol{\Phi}\left(x_{1}, x_{3}, t\right)=\left[\sum_{m} \mathrm{C}_{m} \alpha_{4}^{m} e^{\mathrm{i} k b^{m} x_{3}}\right] \cdot e^{\mathrm{i} k\left(x_{1}-v t\right)} .
\end{aligned}
$$

\section{Electric Potential at Output IDT}

Once the plane wave equation is evaluated for the electric potential wave in the SAW devices, the following analysis can be applied to find out the SAW electric potential at the output IDT. It is assumed that the crystal cut is best set so that the SAW velocity is directed to the $x_{1}$ direction to allow an orthogonal interaction between the SAW and the output IDT. Moreover to simplify the analysis, the acoustic wave is assumed to pass the output IDT once, and interact with no reflections caused by impedance mismatches $[8,13]$. Since the behavior of the propagating waves and the placement of the IDT being periodic, the analysis is carried out for a single period as shown in Fig. 3.

Fig. 3 IDT finger representation for one wavelength with a metalisation ratio of 0.5 . Here $\lambda$ is the wavelength of the SAW

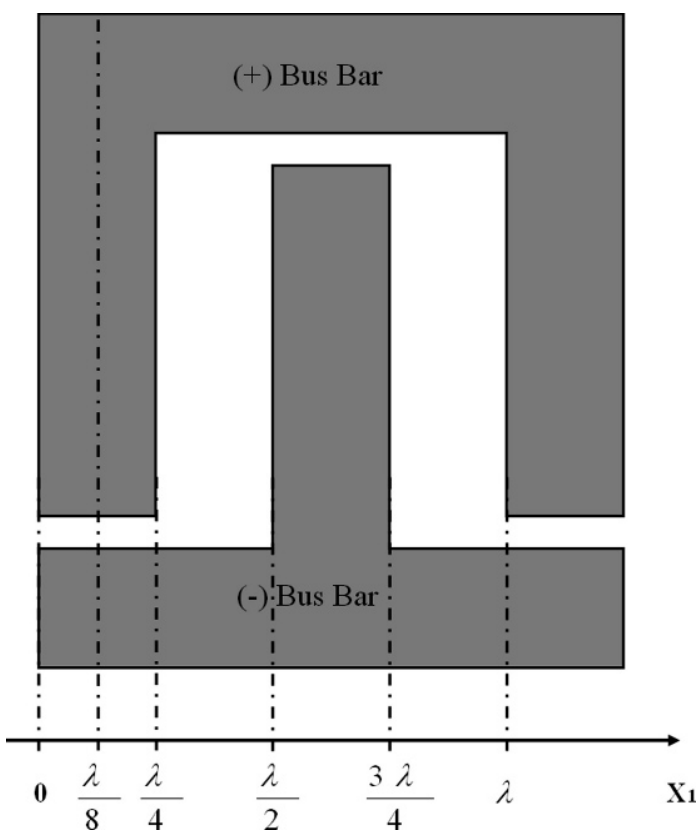


Output IDT fingers are represented as square waves with the period defining wave length of the SAW and the duty cycle defining the finger width [14]. This width is $\lambda / 4$ for a metallisation ratio of 0.5 . For the SAW based electrostatic actuator model, initially a basic model of the IDT configuration was used where each finger is connected to one of two IDT bus bars alternatively as shown in Fig. 3. Since one bus bar is assigned a positive value and the other bus bar is assigned a negative value, each finger will be assigned a positive or negative value determined by the finger's connection to a particular bus bar. At the output IDT, the IDT is considered as a combination of square waves as it was explained above and the SAW is defined as a plane wave as it was shown in Sect. 4. In order to obtain the time response of the surface acoustic wave interaction with the output IDT, a cross-correlation is applied to the SAW and the IDT signals as shown in Fig. 4. As a result the resultant electric potential wave at the output IDT region is a combination of both the electric potential at the IDT and the electric potential at the IDT finger gaps as expressed in (13).

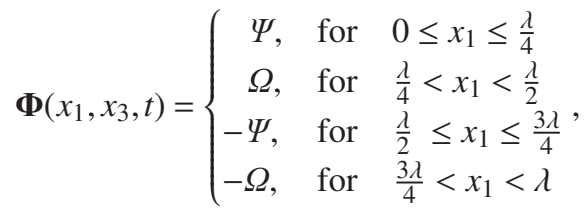

where $\Psi=\boldsymbol{\Phi}\left(\frac{\lambda}{8}, x_{3}, t\right)$ and $\Omega=\boldsymbol{\Phi}\left(x_{1}, x_{3}, t\right)$

$$
\text { for } \boldsymbol{\Phi}\left(x_{1}, x_{3}, t\right)=\left[\sum_{m} \mathrm{C}_{m} \alpha_{4}^{m} e^{\mathrm{i} k b^{m} x_{3}}\right] \cdot e^{\mathrm{i} k\left(x_{1}-v t\right)} \text {. }
$$

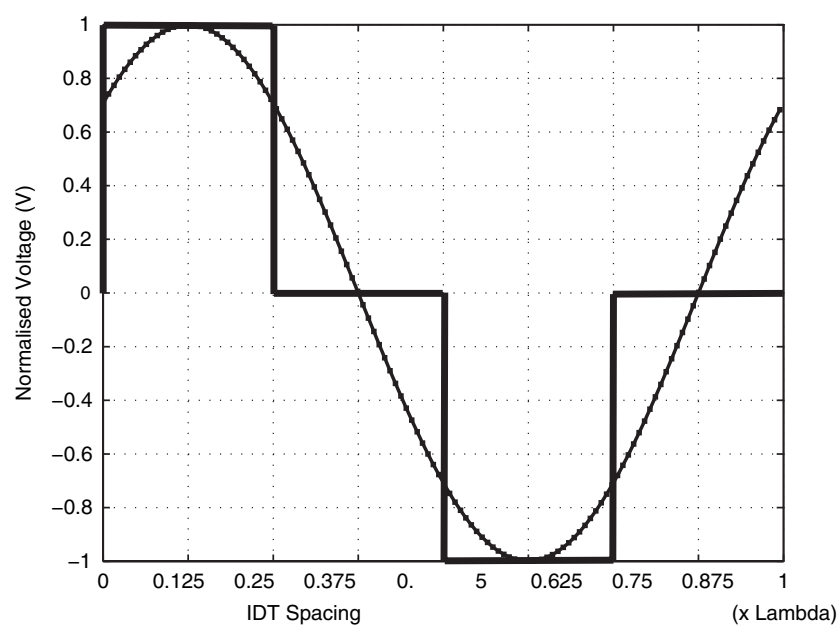

Fig. 4 Correlation between SAW electric potential and the output IDT 


\section{Boundary Condition Analysis}

Weighting coefficients in (11) and (12) need to be determined based on the electric and mechanical boundary conditions applicable for the SAW device based actuator model.

Mechanical boundary conditions: For simplicity, the IDT mass is taken to be negligible so that the mechanical force acting on the SAW substrate can be discarded. Hence the surface is considered be mechanically free. This can be mathematically expressed as

$$
\sum_{j} \mathbf{T}_{3 j}=0 .
$$

Electrical boundary conditions: The surface $\left(x_{3}=0\right)$ where the output IDT is present and the gap between the output IDT fingers were considered separately for electric boundary conditions. As explained in Sect. 5 the electric potential wave at the IDT fingers act as an equipotential time varying wave throughout the positive and negative IDT fingers (does not vary with $x_{1}$ ). Where as the electric potential wave between the fingers still a time varying and a moving wave (varies with $x_{1}$ ). Since the conductive plate is placed at a height $h$ above the output IDT, the electric potential goes to zero at $x_{3}=h$. It should be noted that the electric potential above the surface satisfies Laplace's equation. Also the electric potential and the electric displacement in the $x_{3}$ direction are continuous at the surface $\left(x_{3}=0\right)[6,11]$. These conditions can be mathematically expressed as

$$
\begin{aligned}
& \mathbf{E}\left(x_{3}=0^{-}\right)=\mathbf{E}\left(x_{3}=0\right)=\mathbf{E}\left(x_{3}=0^{+}\right), \\
& \mathbf{D}\left(x_{3}=0^{-}\right)=\mathbf{D}\left(x_{3}=0\right)=\mathbf{D}\left(x_{3}=0^{+}\right),
\end{aligned}
$$

and Laplace's equation can be written as

$$
\boldsymbol{\Phi}_{L}\left(x_{1}, x_{3}, t\right)=\left[A e^{k x_{3}}+B e^{-k x_{3}}\right] \cdot e^{\mathrm{i} k\left(x_{1}-v t\right)} .
$$

The electric potential at $x_{3}=h$ is zero because of the conductive plate. Using this condition, $B$ can be eliminated from (16) and can be rewritten as

$$
\boldsymbol{\Phi}_{L}\left(x_{1}, x_{3}, t\right)=A\left[e^{k x_{3}}-e^{2 k h-k x_{3}}\right] \cdot e^{\mathrm{i} k\left(x_{1}-v t\right)} .
$$

It should be noted that the analysis has to be carried out in two parts, considering the space above the output IDT $\left(0 \leq x_{1} \leq \frac{\lambda}{4} \cup \frac{\lambda}{2} \leq x_{1} \leq \frac{3 \lambda}{4}\right)$ and the space above the output IDT gap $\left(\frac{\lambda}{4}<x_{1}<\frac{\lambda}{2} \cup \frac{3 \lambda}{4}<x_{1}<\lambda\right)$. But the procedure for derivation of $A$ is the same for both the cases. Therefore the approach is shown only for one case.

Considering the plane wave equation (13) for electric potential and (17), and the continuity equation (15), $A$ can be evaluated as follows,

$$
\boldsymbol{\Phi}_{L}\left(x_{1}, 0, t\right)=\boldsymbol{\Phi}\left(x_{1}, 0, t\right),
$$

therefore,

$$
A\left[1-e^{2 k h}\right] \cdot e^{\mathrm{i} k\left(x_{1}-v t\right)}=\left[\sum_{m} \mathrm{C}_{m} \alpha_{4}^{m}\right] \cdot e^{\mathrm{i} k\left(x_{1}-v t\right)},
$$


hence,

$$
A=\frac{\sum_{m} \mathrm{C}_{m} \alpha_{4}^{m}}{\left[1-e^{2 k h}\right]} .
$$

Considering the relationship between the electric displacement and the electric field $\mathbf{D}=\varepsilon_{0} \mathbf{E}$, and (1), a relationship between the electric displacement and the electric potential in the gap can be written as

$$
\mathbf{D}_{3}\left(x_{1}, x_{3}, t\right)=-\varepsilon_{0} \frac{\partial \boldsymbol{\Phi}_{L}\left(x_{1}, x_{3}, t\right)}{\partial x_{3}} .
$$

Using the above, the electric displacement at $x_{3}=0$ can be written as

$$
\mathbf{D}_{3}\left(x_{1}, 0, t\right)=-k \varepsilon_{0} \frac{\sum_{m} \mathrm{C}_{m} \alpha_{4}^{m}\left[1+e^{2 k h}\right]}{\left[1-e^{2 k h}\right]} \cdot e^{\mathrm{i} k\left(x_{1}-v t\right)} .
$$

Another expression for the electric displacement at $x_{3}=0$ is obtained from the equation (6).

$$
\mathbf{D}_{3}\left(x_{1}, 0, t\right)=\sum_{j} \sum_{k} \mathbf{e}_{3 j k} \mathbf{S}_{j k}+\sum_{j} \varepsilon_{3 j}^{S} \mathbf{E}_{j} .
$$

By equating (19) and (20), the relevant electric boundary condition can be obtained and specific values for the weighting coefficients $\mathbf{C}$ can be calculated by solving an eigenvalue problem.

\section{Simulations and Results}

\subsection{Static Analysis}

The performance of the SAW device based electrostatic actuator behavior is simulated using the ANSYS simulation tools [15]. Initially the static analysis is carried out to determine the displacement of the actuator. In order to mimic the effect of the electric potential wave generated at the output IDT of the SAW device, a set of interleaved electrodes are used and every alternative electrode is coupled, so that one set of electrodes act as the positive bus bar and the other as the negative bus bar. Hence the whole SAW device in the microfluidic device is replaced at simulation level. Material properties of silicon are used for the doubly-clamped conductive plate, which in turn acts as the microactuator. The conductive plate dimensions are chosen to be $1000 \mu \mathrm{m} \times 2 \mu \mathrm{m} \times 10 \mu \mathrm{m}(L \times H \times W)$. The gap between the electrodes and the conductive plate $h$ is taken to be $10 \mu \mathrm{m}$ and considered to be filled with air. AC sinusoidal wave with a frequency of $500 \mathrm{MHz}$ and a peak voltage value of 10 volts is used to emulate the electric potential wave at the output IDT (as given in (13)). The conductive plate is connected to ground so that the plate acts as an equipotential surface.

To verify the simulation results, commonly used Rayleigh-Ritz iterative method was used as well. For comparison purposes, displacement versus voltage results are plotted in MATLAB and are shown in Fig. 5. 


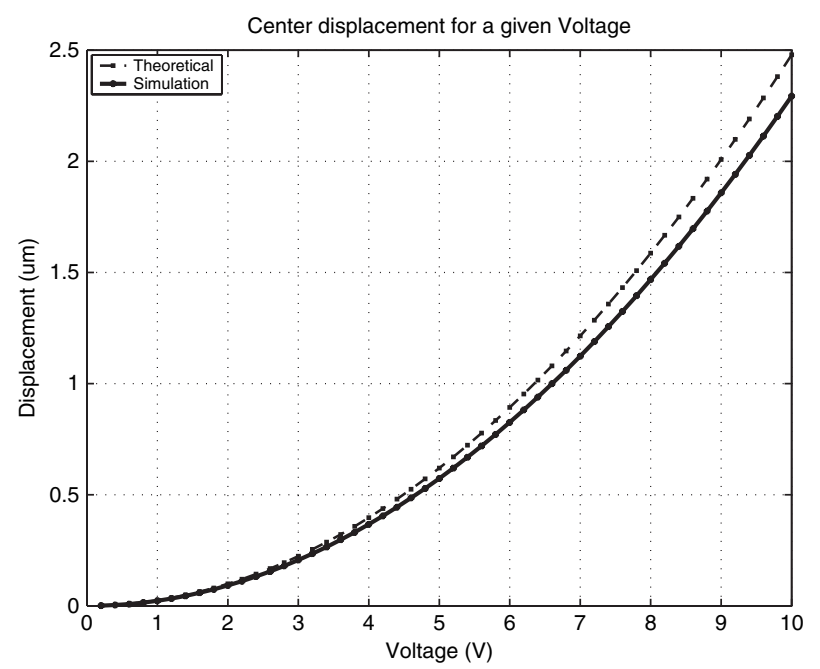

Fig. 5 Comparison of simulated and theoretical results for the SAW actuator. Displacement vs Voltage plot for the mid-beam displacement in the conductive plate actuator above the SAW device

A contour plot obtained from ANSYS simulations which depicts the bending of the actuator, is shown in Fig. 6. The actuator displacement can be optimised by reducing the gap between the conductive plate and the output IDT, reducing the thickness of the conductive plate, and reducing the stress level applied at the actuator by optimising the clamping mechanism.

\subsection{Transient Analysis}

It should be noted that when a conductive beam is subject to a dynamically changing electrostatic field, the displacement behaviour needs to be calculated analytically using an iterative process. At the initial stage, ANSYS based Finite Element Analysis (FEA) method greatly helps to analyse such a dynamic behaviour as the ANSYS solvers are equipped with analytical algorithms. This section depicts the transient simulation results carried out for a conductive plate with the same dimensions mentioned in the static analysis above. Moreover the input electrical signal is similar to the one that was used for the static analysis.

As can be seen from these simulations, the micro displacements are successfully obtained using this method. Figure 7 shows the mid-beam and the quarterbeam displacement variation over the time (250-time-periods). Based on authors' previous work, it was shown that beam displacements up to $2 \mu \mathrm{m}$ can be achieved using SAW device based actuation [16]. After carrying out a few different transient analysis simulations with different end times and comparing the obtained transient displacements with the static displacements achieved, it is proven even 


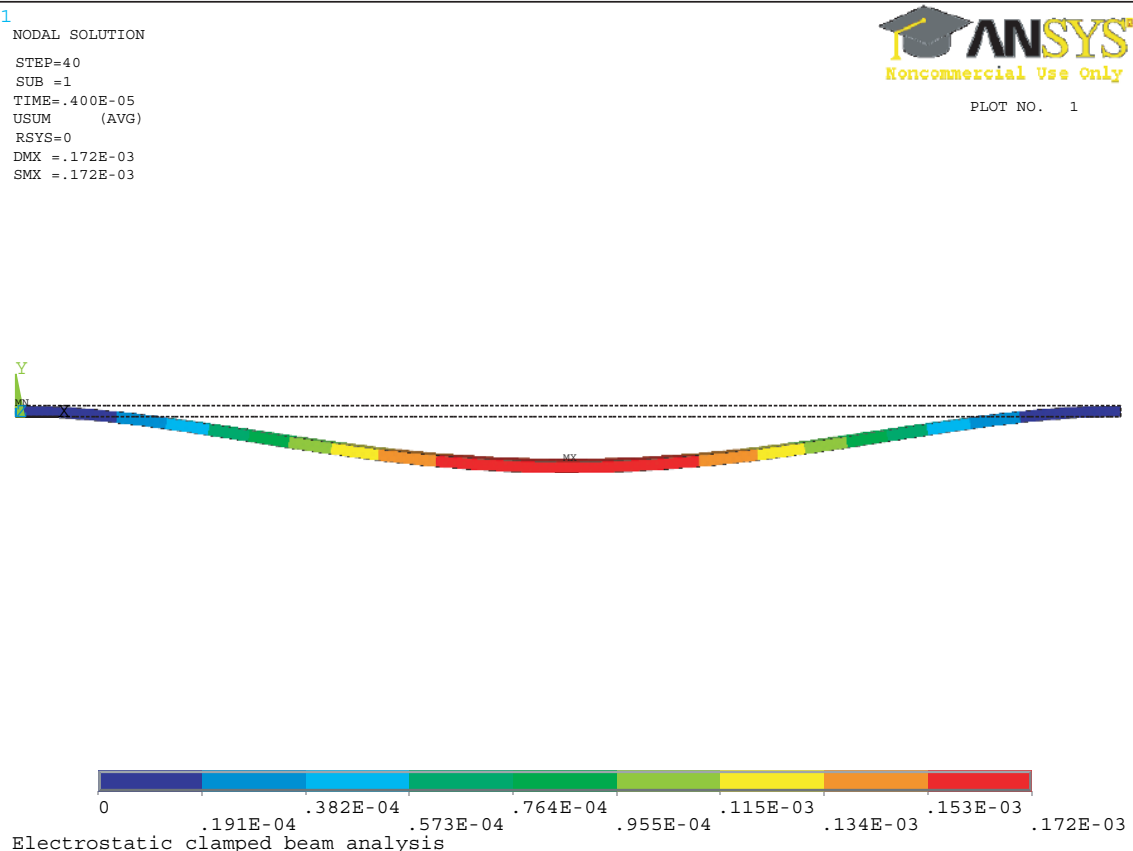

Fig. 6 A side view of the displaced actuator. A thinner actuator $(2 \mu \mathrm{m})$ is analysed to achieve higher displacements [17]

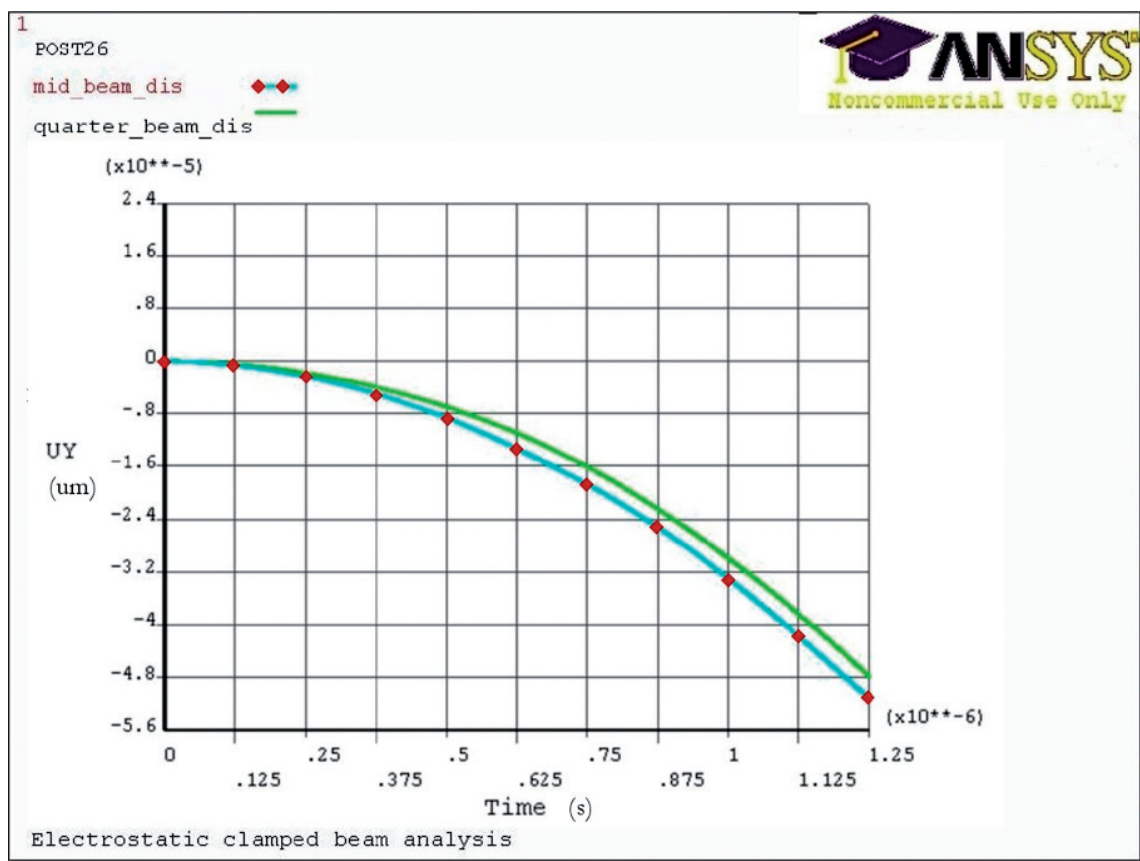

Fig. 7 Displacement vs Time plot of the mid-beam of the conductive plate. Analysis carried out for $250 \times T$, where $T$ is the time period of the SAW signal [17] 




Fig. 8 A Vector plot of the displaced conductive plate obtained after the transient analysis. Vector shows nodal displacements of the conductive plate [17]

after 250-time-periods still the dynamic displacement is not showing any periodic nature but in the process of gaining more displacement. Based on the results obtained so far, it is evident that the actual vibration frequency of the conductive plate is a very much scaled down version of the SAW operating frequency. Figure 8 depicts a vector plot of the displaced conductive actuator. Because of the time varying nature of the electrostatic field that is generated by the output IDTs, the actuator consists of flexural displacement component as it can be seen from the vector plot.

\section{Conclusion}

In this chapter we demonstrated the use of a SAW device to generate microactuations for microfluidic and similar applications. The increasing demand for wireless and low power operated MEMS devices for biomedical applications highlight the requirement of wirelessly interrogated micro devices. Detailed theoretical analysis explaining how the entire SAW device based actuator operation is carried out and boundary conditions applicable for presented design is used to derive the electric potential wave forms, hence the electrostatic field between the SAW device and the conductive plate. Displacement analysis of the conductive actuator is obtained. Static analysis results are generated using the ANSYS simulation tool and compared with the theoretical results obtained by Rayleigh-Ritz method. A good correlation between the theoretical and simulated displacement curves are observed.

Once the static analysis is completed, more importantly a transient analysis is carried out in order to study the dynamic behaviour of the SAW device based electrostatic actuator. This is more substantial in investigating the operating frequency of the conductive plate. Since the SAW frequency is in the range between $100 \mathrm{MHz}-$ $1 \mathrm{GHz}$ it is crucial to verify the effective operating frequency of the conductive plate. Because of the time varying electrostatic field, the operating frequency of the actuator is much less than that of the SAW frequency. 
Acknowledgements The authors would like to thank the Australian Research Council (ARC) and the School of Electrical and Electronic Engineering (University of Adelaide) for the funding and the support provided for this research.

\section{References}

1. Dissanayake D, Tikka A, Al-Sarawi S, Abbott D (2006) Radio frequency controlled microvalve for biomedical applications. In: Proc. SPIE-Smart Materials IV, 6413, Australia, 64130, pp 1-13

2. Varadan V K, Varadan V V (2000) Microsensors, micromechanical systems (MEMS), and electronics for smart structures and systems. Smart Materials and Structures, 9:953-972

3. Wixforth A (2003) Acoustically driven planar microfluidics. Superlattices and Microstructures, 6:389-396

4. Strobl C J, Guttenberg Z V, Wixforth A (2004) A Nano-and pico-dispensing of fluids on planar substrates using SAW. IEEE Transactions on Ultrasonics, Ferroelectrics, and Frequency Control, 51(11):1432-1436

5. Jones I, Ricciardi L, Hall L, Hansen H, Varadan V, Bertram C, Maddocks S, Enderling S, Saint D, Al-Sarawi S, Abbott D (2007) Wireless RF communication in biomedical applications. Smart Materials and Structures, 17015050 (10pp)

6. Subramanian H, Varadan V K, Varadan V V, Vellekoopz M J (1997) Design and fabrication of wireless remotely readable MEMS based microaccelerometers. Smart Materials and Structures, 6(6):730-738

7. Milstein L B, Das P (1979) Surface acoustic wave devices. IEEE Communications Magazine, 17:25-33

8. Gardner J W, Varadan V K, Awadelkarim O O (2001) Microsensors, MEMS, and Smart Devices. Tsinghua University Press, Beijing

9. Gantner A, Hoppe R H W, Köster D, Siebert K G, Wixforth A (2005) Numerical simulation of piezoelectrically agitated surface acoustic waves on microfluidic biochips. http://www.opusbayern.de/uni-augsburg/volltexte/2005/142/, visited on 14/02/2008

10. Kannan T (2006) Finite element analysis of surface acoustic wave resonators. MA thesis, University of Saskatchewan

11. Maugin G A (1985) Nonlinear electromechanical effects and applications. World Scientific Publishing Co Pty Ltd, Singapore

12. Adler E L (2000) Bulk and surface acoustic waves in anisotropic solids. International Journal of High Speed Electronics and Systems, 10(3):653-684

13. Ippolito S J, Kalantar-zadeh K, Wlodarski W, Powell D A (2002) Finite-element analysis for simulation of layered SAW devices with XY LiNbO3 substrate Proc. In: SPIE-Smart Structures, Devices, and Systems, 4935, Australia, pp 120-131

14. Skinner J L, Cardinale G F, Talin A A, Brocato R W (2006) Effect of critical dimension variation on SAW correlator energy. IEEE Transactions on Ultrasonics, Ferroelectrics and Frequency Control, 53(2):497-501

15. ANSYS Incorporation. Home page, http://www.ansys.com/, visited on 14/02/2008

16. Dissanayake D, Al-Sarawi S, Abbott D (2007) Surface Acoustic Wave Device Based Electrostatic Actuator for Microfluidic Applications. In: Proc. 2nd International Conference on Sensing Technology, Palmerston North, New Zealand, pp 381-386

17. Dissanayake D W, Al-Sarawi S, Abbott D (2007) Surface Acoustic Wave Device based wireless passive microvalve for microfluidic applications. In: Proc. SPIE-BioMEMS and Nanotechnology III, 6799, 67990H pp 1-10 